\title{
Automatic generation of symmetry-breaking constraints
}

\author{
Leo Liberti \\ LIX, École Polytechnique, F-91128 Palaiseau, France \\ liberti@lix.polytechnique.fr
}

\begin{abstract}
Solution symmetries in integer linear programs often yield long Branch-and-Bound based solution processes. We propose a method for finding elements of the permutation group of solution symmetries, and two different types of symmetry-breaking constraints to eliminate these symmetries at the modelling level. We discuss some preliminary computational results.
\end{abstract}

\section{Introduction}

We consider a Mixed Integer Linear Program (MILP) in the following form:

$$
\left.\begin{array}{rl}
\min c^{\top} x & \\
A x & \leq b \\
x & \in\left[x^{L}, x^{U}\right] \\
\forall i \in Z \quad x_{i} & \in \mathbb{Z} .
\end{array}\right\}
$$

where $c, x, x^{L}, x^{U} \in \mathbb{R}^{n}, b \in \mathbb{R}^{m}, A$ is a real $m \times n$ matrix and $Z \subseteq\{1, \ldots, n\}$. Throughout the paper, elements of groups are represented by means of permutations of either the column or the row space; permutations on the row space are denoted by left multiplication, and permutations on the column space by right multiplication. Because a solution $x$ of (1) has as many elements as the columns of $A$, a permutation $\pi$ on $x$ is likened to a permutation of the column space, and hence denoted by right multiplication $x \pi$.

Problems (1) having many symmetries are known to be very difficult to solve to global optimality with Branch-and-Bound (BB) techniques. These converge slowly in presence of symmetries because many leaf nodes in the BB tree may contain (symmetric) global optima: hence, no node in the paths leading from the root to these leaf nodes can ever be pruned. Despite the practical difficulties given by solution symmetries, group-theoretical methods in mathematical programming did not have an impressively wide diffusion over the years, and may be classified in three broad categories: (a) the abelian group approach proposed by Gomory to writing integer feasibility conditions for $x$; (b) symmetry-breaking techniques for specific problems, whose symmetry group can be computed in advance; (c) general-purpose symmetry group computations and symmetry-breaking techniques implemented via branching strategies and local cuts in a typical BB solution algorithm. 
Category (a) was established by R. Gomory [7]: given a basis $B$ of the constraint matrix $A$, it considers the (abelian) group $\mathscr{G}=\mathbb{Z}^{n} /\langle\operatorname{col}(B)\rangle$, where $\mathbb{Z}^{n}$ is the additive group of integer $n$-sequences and $\langle\operatorname{col}(B)\rangle$ is the additive group generated by the columns of the (nonsingular) matrix $B$. We consider the natural group homomorphism $\varphi: \mathbb{Z}^{n} \rightarrow \mathscr{G}$ with $\operatorname{ker} \varphi=\langle\operatorname{col}(B)\rangle$ : letting $\left(x_{B}, x_{N}\right)$ be a basic/nonbasic partition of the decision variables, we apply $\varphi$ to the standard form constraints $B x_{B}+N x_{N}=b$ to obtain $\varphi\left(B x_{B}\right)+\varphi\left(N x_{N}\right)=\varphi(b)$. Since $\varphi\left(B x_{B}\right)=0$ if and only if $x_{B} \in \mathbb{Z}^{n}$, setting $\varphi\left(N x_{N}\right)=\varphi(b)$ is a necessary and sufficient condition for $x_{B}$ to be integer feasible. Gomory's seminal paper gave rise to further research, among which $[22,1]$

Category (b) is possibly the richest in terms of number of published papers. Many types of combinatorial problems exhibit a certain amount of symmetry. Symmetries are usually broken by means of specific branching techniques (e.g. [16]), appropriate global cuts (e.g. [21]) or special formulations [11, 2] based on the problem structure. The main limitation of the methods in this category is that they are difficult to generalize and/or to be rendered automatic.

Category (c) contains two main research streams. The first was established by F. Margot in the early 2000s [14,15], and is applicable to problems in general form (1) where $x^{L}=0, x^{U}=1$, i.e. Binary Linear Programs (BLPs). Margot defines the symmetry group of a BLP as:

$$
\left\{\pi \in S_{n} \mid c^{\top} \pi=c^{\top} \wedge \exists \sigma \in S_{n}(\sigma b=b \wedge \sigma A \pi=A)\right\},
$$

or, in other words, all relabellings of problem variables for which the objective function and constraints are the same. The symmetry group (2) is used to derive effective BB pruning strategies by means of isomorphism pruning and isomorphism cuts local to some selected BB tree nodes (Margot extended his work to general integer variables in [17]). Stronger results of the same type can be obtained for covering and packing problems [20], for these have an objective function vector $c=(1, \ldots, 1)$ and a RHS vector $b=(1, \ldots, 1)$ fixed by all elements of $S_{n}$ and $S_{m}$ respectively, and their constraint matrix is 0-1.

The second was established by V. Kaibel and M. Pfetsch in 2007 [9]. Symmetries in the column space (i.e. permutations of decision variables) of binary ILPs having 0-1 constraint matrices are shown to affect the quality of the linear programming bound. Limited only to permutations in cyclic and symmetric group, complete descriptions of orbitopes are provided by means of linear inequalities. Let $x^{\prime}$ be a point in $\{0,1\}^{n}$ (the solution space), with $n=p q$, so that we can arrange the components of $x^{\prime}$ in a matrix $C$. Given a group $G$ and $\pi \in G$, for all 0-1 $p \times q$ matrices $C$ let $C \pi$ be the matrix obtained by permuting the columns of $C$ according to $\pi$. Let $G \cdot C$ be the orbit of $C$ under the action of all $\pi \in G$, $\overline{G \cdot C}$ be the lexicographically maximal matrix in $G \cdot C$ (ordering matrices by rows first) and $\mathcal{M}_{p q}^{\max }(G)$ be the set of all $\overline{G \cdot C}$. Then the full orbitope associated with $G$ is $\operatorname{conv}\left(\mathcal{M}_{p q}^{\max }(G)\right)$. Inspired by the work on orbitopes, E. Friedman very recently proposed a similar but extended approach leading to fundamental domains [5]: given a feasible polytope $X \subseteq[0,1]^{n}$ with integral extreme points and a group $G$ acting as an affine transformation on $X$ (i.e. for all $\pi \in G$ there 
is a matrix $A \in G L(n)$ and an $n$-vector $d$ such that $\pi x=A x+d$ for all $x \in X)$, a fundamental domain is a subset $F \subset X$ such that $G F=X$.

The Constraint Programming (CP) community is also concerned with symmetries and some of the results in the CP literature can be extended to mathematical programming (see [3] for a good introduction).

The present work belongs to category (c): it proposes general-purpose methods for identifying (some) solution symmetries and restrict the feasible region so that it does not contain all representatives per equivalence class. This paper contributes two ideas: (i) breaking the same type of symmetries described in $[16,14,15,17]$ at the modelling instead of the algorithmic level (whereas Margot proposes symmetry breaking methods local to each node of the BB tree, we discuss global symmetry breaking constraints which can be added to the original formulation); and (ii) computing symmetries automatically instead of assuming them as given. In Section 2 we propose symmetry breaking constraints derived from cycles; Section 3 describes a mathematical program whose solution encodes a permutation of the symmetry group; in Section 4 we discuss some practical strategies for exploiting the proposed symmetry breaking constraints and some preliminary computational results.

\subsection{Notation}

For a group $G \leq S_{n}$ and a set $X$ of row vectors, $X G=\{x g \mid x \in X \wedge g \in G\}$; if $Y$ is a set of column vectors, $G Y=\{g y \mid y \in Y \wedge g \in G\}$. If $X=\{x\}$, we denote $X G$ by $x G$ (and similarly for $Y$ ). For a mathematical program $P$ we let $\mathcal{F}(P)$ be the feasible region of $P$ and $\mathcal{G}(P)$ be the set of (global) optima of $P$. For $x \in \mathbb{R}^{n}$, we let $\operatorname{ran}(x)=\left\{a \in \mathbb{R} \mid \exists j \leq n\left(x_{j}=a\right)\right\}$ be the range of $x$. All groups considered in this paper are finite.

\section{Theoretical results}

\subsection{Efficiency of symmetry breaking constraints}

We let $S_{n}$ be the symmetric group of order $n \in \mathbb{N}$. For a set $X \subseteq \mathbb{R}^{n}$, a group $G \leq S_{n}$ and $x, y \in X$, we define an equivalence relation $x \sim_{G} y \Leftrightarrow \exists \pi \in G(x \pi=$ $y$ ). The relation $\sim_{G}$ partitions $X$ into a set $\mathcal{E}(G, X)$ of equivalence classes (each of finite cardinality) such that $X=\bigcup_{Y \in \mathcal{E}(G, X)} Y$ (the cardinality of $\mathcal{E}(G, X)$ itself need not be finite or even countable).

\subsection{Definition}

A linear constraint $d x \leq d_{0}$ with $\left(d, d_{0}\right) \in \mathbb{R}^{n+1}$ is symmetry breaking with respect to $G$ and $X$ if for all $Y \in \mathcal{E}(G, X)$ there are $\bar{x} \neq \bar{y} \in Y$ s.t. $d \bar{x} \leq d_{0}$ and $d \bar{y}>d_{0}$. The constraint is symmetry breaking of order $\ell$ if there is at least one equivalence class $Y \in \mathcal{E}(G, X)$ in which there are exactly $\ell-1$ points $y$ s.t. $d \bar{y}>d_{0}$. The constraint is maximally symmetry breaking if there is at least one equivalence class $Y \in \mathcal{E}(G, X)$ for which it is symmetry breaking of order $|Y|$. 
Defn. 2.1 can easily be extended to systems of constraints. Supposing $X \subseteq \mathbb{Z}^{n}$, symmetry breaking constraints are not in general valid cuts for any linear polyhedron containing $X$, because they may also cut off some integral points. However, they guarantee feasibility of at least one integral point per equivalence class. Adding appropriate symmetry breaking constraints to $P$ results in a reformulation of the narrowing type [13], i.e. a reformulation $Q$ of $P$ with a map $\psi: \mathcal{F}(Q) \rightarrow \mathcal{F}(P)$ such that $\psi(\mathcal{G}(Q)) \subseteq \mathcal{G}(P)$ [12]. Notice that symmetry breaking constraints of order $\ell \leq 1$ do not break any symmetry at all, as they do not separate any point in any equivalence class of $\mathcal{E}(G, X)$.

\subsection{Symmetry groups associated to a MILP}

We consider symmetries that leave various properties of $P$ invariant.

\subsection{Definition}

The set

$$
G^{*}=\left\{\pi \in S_{n} \mid \forall x \in \mathcal{G}(P)(x \pi \in \mathcal{G}(P))\right\}
$$

of automorphisms of $\mathcal{G}(P)$ is called the solution symmetry group of $P$. The set

$$
\tilde{G}=\left\{\pi \in S_{n} \mid \forall x \in \mathcal{F}(P)(x \pi \in \mathcal{F}(P))\right\}
$$

of automorphisms of $\mathcal{F}(P)$ is called the feasible symmetry group of $P$

It is easy to show that $\tilde{G}, G^{*}$ are both subgroups of $S_{n}$, and that $G^{*} \leq \tilde{G}$. Next, we extend (2) to formulation (1).

\subsection{Definition}

The set

$$
\begin{aligned}
G_{P}= & \left\{\pi \in S_{n} \mid \forall a \in\left\{c, x^{L}, x^{U}\right\}\right. \\
& \left.\left(a^{\top} \pi=a^{\top}\right) \wedge Z \pi=Z \wedge \exists \sigma \in S_{n}(\sigma b=b \wedge \sigma A \pi=A)\right\}
\end{aligned}
$$

of permutations that fix the problem formulation is called the problem symmetry group of $P$.

It is equally easy to show that $G_{P}$ is a subgroup of $S_{n}$. The following useful result states that problem symmetries are solution symmetries.

\subsection{Proposition}

$G_{P} \leq G^{*}$.

\subsection{Symmetry breaking constraints from disjoint cycles}

Let $R$ be the relaxation of $P$ obtained by removing the constraints $A x \leq b, \bar{X}=$ $\mathcal{F}(R)$ and $X^{*}=\mathcal{G}(R)$. Let $\sigma=\left(\sigma_{1}, \ldots, \sigma_{k}\right)$ be a cycle of length $1<k \leq n$ in $S_{n}$. For any $x \in \mathbb{R}^{n}$, let $x[\sigma]=\left(x_{\sigma_{1}}, \ldots, x_{\sigma_{k}}\right)$, and assume that $x[\sigma]$ are constrained to be integer. Let $\bar{G} \leq S_{n}$ be the group of all permutation automorphisms of $\bar{X}$. 
If $\sigma \in \bar{G}$, for all $x \in \bar{X}$ and $j \leq k$ we have $x_{\sigma_{1}}^{L} \leq x[\sigma]_{j} \leq x_{\sigma_{1}}^{U}$, i.e. there is a unique number of values $\chi$ that all variables in $x[\sigma]$ can take. We let $\bar{\chi}$ be the row vector whose $j$-th component is $\chi^{k-j}$ for all $j \leq k$. Consider the following constraints, often cited in the literature for symmetry breaking purposes [21, 10]:

$$
\begin{aligned}
\forall 1 \leq h \leq & k-1 \quad \bar{\chi}\left(x[\sigma]-x^{L}[\sigma]\right) \leq \bar{\chi}\left(x[\sigma]-x^{L}[\sigma]\right) \sigma^{h} \\
\Rightarrow \sum_{j=1}^{k} \chi^{k-j}\left(x_{\sigma_{j}}-x_{\sigma_{j}}^{L}\right) & \left.\leq \sum_{j=1}^{k} \chi^{k-j}\left(x_{\sigma^{h}\left(\sigma_{j}\right)}-x_{\sigma^{h}\left(\sigma_{j}\right)}^{L}\right)\right) .
\end{aligned}
$$

\subsection{Proposition}

Let $\sigma=\left(\sigma_{1}, \ldots, \sigma_{k}\right)$ be a cycle of length $k \leq n$ in $\bar{G}$. Then constraints (6) are maximally symmetry breaking w.r.t. $\langle\sigma\rangle, \bar{X}$.

The practical trouble with (6) is their well-known poor scaling, as the values of the coefficients are of different orders of magnitude.

Next, we consider some well-scaled (though less effective) symmetry breaking constraints.

\subsection{Proposition}

Let $\sigma=\left(\sigma_{1}, \ldots, \sigma_{k}\right)$ be a cycle of length $k \leq n$ in $\bar{G}$. For all $x \in \bar{X}$ with $|\operatorname{ran}(x[\sigma])|=\ell$,

$$
\forall 2 \leq j \leq k \quad x_{\sigma_{1}} \leq x_{\sigma_{j}}
$$

are symmetry breaking constraints of order $\ell$.

Notice that if $\ell=k$, then by Prop. 2.6 (7) are symmetry-breaking constraints of order $k$; furthermore, if $\ell=k$ the vector $x \in \mathbb{R}^{n}$ having distinct components $x[\sigma]$ gives rise to an equivalence class $x\langle\sigma\rangle$ of cardinality $k$, so (7) are maximally symmetry breaking. If $\ell=1$ then (7) do not break any symmetry (remark after Defn. 2.1) but then again if $\ell=1$ it means that $x[\sigma]=(a, \ldots, a)$ for some $a \in \mathbb{R}$, so $|x\langle\sigma\rangle|=1$, which means there are no symmetric solutions complicating the solution process. The most likely case is that $x[\sigma] \in\{0,1\}^{k}$ and $\ell=2$ : this is unfortunate as this situation provides the weakest case of Prop. 2.6. We stress, however, that symmetry breaking constraint of order $\ell$ will cut away at least (not exactly) $\ell-1$ symmetric solutions.

\subsection{Example}

Let $x=(0,1,1,1)$, and $\sigma=(1,2,3,4)$. Then $x\langle\sigma\rangle=\{(0,1,1,1),(1,0,1,1)$, $(1,1,0,1),(1,1,1,0)\}$. Since $|\operatorname{ran}(x)|=|\{0,1\}|=2$, constraints (7) are symmetry breaking of order 2 . However, exactly 3 elements of $x\langle\sigma\rangle$ are cut off by (7) (i.e. all elements of $x\langle\sigma\rangle \backslash\{x\})$. Taking $x=(0,0,0,1)$, on the other hand, results in (7) only cutting off $x \sigma=(1,0,0,0)$ according to Prop. 2.6.

The main insight given by Example 2.7 is that if we make the assumption that optimal solutions of binary problems will contain on average as many $0 \mathrm{~s}$ as $1 \mathrm{~s}$ on components indexed by $\sigma$, we can expect (7) to cut away $\lfloor k / 2\rfloor$ symmetric 
solutions even though $\ell=2$. Another insight is that if we suspect optimal solutions to have a large number of components attaining small values of the range, we might want to change the $\leq$ relation in (7) to a $\geq$ relation to increase the number of cut-off symmetric solutions (modifying the inequality relation in (7) to $x_{\sigma_{1}} \geq x_{\sigma_{j}}$ only requires a trivial change to the proof).

\section{$3 \quad$ Finding symmetries}

Let

$$
\begin{aligned}
\hat{G}_{P}=\left\{(\sigma, \pi) \in S_{m} \times S_{n} \mid \forall a \in\left\{c, x^{L}, x^{U}\right\}\right. \\
\\
\left.\left(a^{\top} \pi=a^{\top}\right) \wedge Z \pi=Z \wedge(\sigma b=b \wedge \sigma A \pi=A)\right\} .
\end{aligned}
$$

It is easy to see that the projection of $\hat{G}_{P}$ on the second component $S_{n}$ is equal to $G_{P}$. For $q \in \mathbb{N}$, Let $\vartheta: S_{q} \rightarrow G L(q)$ be the regular (faithful) permutation matrix representation of elements of $S_{q}$, i.e. for $\pi \in S_{q}, \vartheta(\pi)$ is a doubly stochastic invertible matrix with entries in $\{0,1\}$, such that for any row vector $v \in \mathbb{R}^{n}$, $v \pi=v \theta(\pi)$. We can then write the condition of (8) in terms of products of vectors and matrices.

We consider decision variables: $\sigma_{i h}$, the $(i, h)$-th element of the matrix $\vartheta(\sigma)$ for all $i, h \leq m, \sigma \in S_{m}$; and $\pi_{j k}$, the $(j, k)$-th element of the matrix $\vartheta(\pi)$ for all $j, k \leq n, \pi \in S_{n}$. Let $z \in\{0,1\}^{n}$ be the indicator vector of $Z$, such that $z_{j}=1 \Leftrightarrow j \in Z$, and let $\Gamma(P)$ be the set of binary values of $\sigma, \pi$ defined by the following constraints:

$$
\begin{aligned}
\forall j \leq n \quad \sum_{k \leq n} x_{k}^{L} \pi_{j k}=x_{j}^{L} & \wedge \sum_{k \leq n} x_{k}^{U} \pi_{j k}=x_{j}^{U} \\
\forall j \leq n \sum_{k \leq n} c_{k} \pi_{j k}=c_{j} & \wedge \sum_{k \leq n} z_{k} \pi_{j k}=z_{k} \\
\forall i \leq m, j \leq n \sum_{h \leq m} \sigma_{i h} A_{h j} & =\sum_{k \leq n} A_{i k} \pi_{k j} \\
\forall i \leq m \sum_{h \leq m} \sigma_{i h} b_{h} & =b_{i} \\
\forall j \leq n \quad \sum_{k \leq n} \pi_{k j}=1 & \wedge \sum_{k \leq n} \pi_{j k}=1 \\
\forall i \leq m \sum_{h \leq m} \sigma_{i h}=1 & \wedge \sum_{h \leq m} \sigma_{h i}=1 \\
\forall j, k \leq n \quad \pi_{k j} & \in\{0,1\} \\
\forall i, h \leq m \quad \sigma_{i h} & \in\{0,1\} .
\end{aligned}
$$

It is easy to show that $\Gamma(P)=\left\{(\vartheta(\sigma), \vartheta(\pi)) \mid(\sigma, \pi) \in \hat{G}_{P}\right\}$. In order to exclude the identity from $\Gamma(P)$, we also add the constraint:

$$
\sum_{j \leq n} \pi_{j j} \leq n-1
$$


Since by Sect. 4 we look for long cycles, we arbitrarily choose an index $j^{\prime} \leq n$ which is likely to belong to a long cycle in some permutation of $G_{P}$ (this choice should be based on the block structure of $A$ ) and minimize the following objective function, which ensures that we select a permutation moving $j^{\prime}$ :

$$
\min \pi_{j^{\prime} j^{\prime}}
$$

We call the problem of minimizing (18) subject to (9)-(17) the Feasible PeRmutation Program associated to $P$ w.r.t. $j^{\prime}$, denoted by $\operatorname{FPP}\left(P, j^{\prime}\right)$.

\subsection{Proposition}

If $\operatorname{FPP}\left(P, j^{\prime}\right)$ is infeasible, then $G_{P}=\{e\}$.

Although solving the full $\operatorname{FPP}\left(P, j^{\prime}\right)$ may be more CPU-intensive than solving the original problem, various improvements based on the block structure of the constraints in $\Gamma(P)$ are possible. A promising one consists in solving relaxations of the FPP where (11) only fix certain rows or blocks, and verifying later than the solution is valid in the general problem. Computational experience shows that although the linear relaxation of the FPP may be fractional, solutions to the FPP are mostly found at the root node of the CPLEX [8] BB tree after cuts addition.

\section{Practical solution strategies}

Our strategy for solving (1) consists in seeking permutations of $G_{P}$ having long cycles in their disjoint cycle representation, and add symmetry breaking constraints (6) or (7). In this section we restrict the discussion to the well-scaled constraints (7) but the same ideas can be (and were) applied to (6) too.

Ideally, we would like to be able to add symmetry-breaking constraints (7) for all disjoint cycles in all generators of $G_{P}$. This, however, may lead to infeasibility, as Example 4.1 shows.

\subsection{Example}

Suppose $\mathcal{G}(P)=\{(0,1,1,0),(1,0,0,1)\}$ and $G_{P}=\{e,(1,2)(3,4)\}$. Then both (6) and (7) would imply $x_{1} \leq x_{2}$ and $x_{3} \leq x_{4}$, which are satisfied by no point in $\mathcal{G}(P)$.

The trouble arises because for a cycle $\sigma$, constraints (7) arbitrarily decide that $x_{\sigma_{1}}$ is the component of $x[\sigma]$ having minimum value. At the modelling level, this is similar to the main drawback of the algorithm proposed in [14]: "the branching variable cannot be chosen freely, but always has to be the non-fixed variable with smallest index" ([15], p. 3-4). However, since at the modelling level there is no knowledge of what variables are fixed, (7) can only be imposed for one single cycle. A promising strategy is that of selecting the longest cycle $\sigma$ from the set of all disjoint cycles in all permutations of $G_{P}$. In general, we can change the arbitrary choice of minimum component for any $i \leq|\sigma|$ (the cycle length), and we denote BreakSymm2 $(P, i)$ the reformulated problem $P$ with $(7)$ adapted 
to $\sigma$ and $i$ as added constraints. It is easy to show that $\operatorname{BrEAKSymm} 2(P, i)$ is a valid narrowing for all cycles $\sigma$ and $i \leq|\sigma|$, albeit one that is still subject to an arbitrary choice. We circumvent this by introducing continuous variables $y_{i}^{\sigma} \geq 0$ whose value is exactly 0 only if $x_{\sigma_{i}}$ is the minimum element of $\sigma$, and reformulating (7) as follows:

$$
\begin{array}{r}
\forall i, j \leq|\sigma|, j \neq i \quad x_{\sigma_{i}}-x_{\sigma_{j}} \leq y_{i}^{\sigma} \\
\sum_{i \leq|\sigma|} y_{i}^{\sigma} \leq|\sigma|-1 .
\end{array}
$$

Constraints (19) express the fact that there may be indices $i$ for which $x_{\sigma_{i}}$ is minimum in $x[\sigma]$, and (20) say that there is at least one such $i$, thus yielding a narrowing BREAKSymm2 $(P)$ that is independent of of the choice of $i$. We remark that an aggregated version of (19), when combined with (20), produces a narrowing $Q^{\prime}$ such that $\mathcal{F}\left(Q^{\prime}\right)=\mathcal{F}(\operatorname{BrEAKSymm} 2(P))$ :

$$
\forall i \leq k \quad(|\sigma|-1) x_{\sigma_{i}}-\sum_{j \neq i} x_{\sigma_{j}} \leq(|\sigma|-1) y_{i}^{\sigma} .
$$

Although in general aggregated constraints tend to produce slacker linear relaxations [19], we mention (21) here because in the tested instances they usually improve CPU times.

Since (19)-(20) together simply express the fact that there is a minimum component in each $x[\sigma]$, and this sentence is true for each disjoint cycle and each permutation in $G_{P}$, it follows that (19)-(20) can be added to $P$ for each cycle $\sigma$ appearing in the set of disjoint cycles over all permutations of $G_{P}$, yielding a valid narrowing denoted by BREAKSymm $2 \operatorname{All}(P)$. This, however, adds several variables and constraints to $P$, which implies that the size of the solution set increases, and each linear relaxation costs more in terms of CPU time; moreover, although BREAKSymm2 $\operatorname{All}(P)$ is a valid narrowing of $P$, the relaxation of BREAKSymm2ALL $(P)$ need not be strictly tighter than that of $P$.

\subsection{Computational experiments}

The results we report should be treated as preliminary experiments rather than full computational results. Although the whole software structure is in place and the process of finding symmetries and then solutions of MILPs has been made fully automatic (by means of several different software packages, some developed on purpose and some off-the-shelf such as AMPL [4], CPLEX [8] and GAP [6]), finding elements of $G_{P}$ automatically is still too costly in terms of CPU time. Moreover, because of the small size of problems for which it was possible to automatically compute symmetries, the addition of constraints and variables and consequent higher cost at each $\mathrm{BB}$ node offset the advantages of the narrowing as regards CPU time.

We employed the test set as described in Table 1. Instance is the instance name, Source lists the instance library or citation where the instance appears, 
Integers and Constraints report the number of integer variables and constraints respectively, Size lists the size of the instance in bytes, and Infeasible is 1 if the instance is infeasible. Table 2 reports the results. Column Group contains the

\begin{tabular}{|l||c|r|r|r|r|}
\hline Instance & Source & Integers & Constraints & Size & Infeasible \\
\hline enigma & {$[18]$} & 100 & 21 & 5616 & 0 \\
jgt18 & {$[11]$, p. 413} & 132 & 105 & 9399 & 1 \\
oa66234 & {$[17]$, Table 1} & 64 & 42 & 5176 & 0 \\
oa67233 & {$[17]$, Table 1} & 128 & 64 & 12153 & 0 \\
oa76234 & {$[17]$, Table 1} & 64 & 42 & 5176 & 0 \\
of sub9 & {$[11]$, p. 413} & 203 & 92 & 13157 & 1 \\
stein27 & {$[18]$} & 27 & 118 & 5789 & 0 \\
sts27 & {$[15]$, p. 17} & 27 & 117 & 5612 & 0 \\
\hline
\end{tabular}

Table 1. Test set description.

subgroup of $G_{P}$ found automatically by repeatedly solving a version of the FPP with random coefficients on the objective function (we call this the "randomized FPP procedure"); the group descriptions are non-unique, as there are many possible semi-direct product types $\ltimes$, see e.g. sts27 and stein27. Longest contains the size of the longest cycle in the group generators, which is used to formulate SymmBreak2 $(P) . N$ is the number of nodes in the BB tree of the original problem $P$, and $N^{\prime}$ is the number of nodes in the BB tree of BreakSymm2 $(P)$. The instances were solved by CPLEX 10.1 [8] on one core of a 32 bit Intel Core Duo 1.2 $\mathrm{GHz}$ with 1.5GB RAM running Linux.

\begin{tabular}{|l||l|r|r|r|}
\hline Instance & Group & Longest & $N$ & $N^{\prime}$ \\
\hline enigma & $C_{2}$ & 2 & 3321 & $\mathbf{2 6 9}$ \\
jgt18 & $C_{2} \times S_{4}$ & 6 & $\mathbf{5 7 3}$ & 1300 \\
oa66234 & $S_{3}$ & 2 & 0 & 0 \\
oa67233 & $C_{2} \times S_{4}$ & 6 & 6 & $\mathbf{0}$ \\
oa76234 & $S_{3}$ & 2 & 0 & 0 \\
ofsub9 & $C_{3} \times S_{7}$ & 21 & 1111044 & $\mathbf{9 8 0 4 8 5}$ \\
stein27 & $\left(\left(C_{3} \times C_{3} \times C_{3}\right) \ltimes P S L(3,3)\right) \ltimes C_{2}$ & 24 & $\mathbf{1 0 8 4}$ & 1843 \\
sts27 & $\left(\left(C_{3} \times C_{3} \times C_{3}\right) \ltimes P S L(3,3)\right) \ltimes C_{2}$ & 26 & 1317 & $\mathbf{9 6 8}$ \\
\hline
\end{tabular}

Table 2. Results of the computational experiments. The last column refers to SymmBreak2 $(P)$. The group descriptions were computed by GAP [6].

Remarks on the experiments.

- Although the instance set is definitely still too small to draw significant conclusions (work is ongoing to enlarge it), the encouraging result is that 
there was an improvement on the only really difficult instance (of sub9): even more so as, it being infeasible, $\mathrm{BB}$ performance is poor because there are many fewer prunings than with feasible ones (no upper bounding objective function value is ever available).

- The extensions of Sect. 4 applied to (6) did not yield good results due to the increased constraint matrix density and bad scaling.

- Using the straight version of (6) and (7) (with the arbitrary choice on the chosen longest cycle orbit representative) sometimes decreases $N^{\prime}$ so that even the CPU times are improved, depending on the arbitrary choice; in such cases, (6) were better than (7) as expected.

- Instance stein27 is like sts27 but with an added cardinality constraint (sum of all variables $\geq 13$, a constraint which is inactive on all optimal solutions); this (small) difference in problem formulation caused the randomized FPP procedure to find different permutations (although leading to the same group description, the exact group structure is different) and hence to different performance.

- Sometimes long "easy" cycles are overlooked, such as in the case of oa66234 and oa76234: the group is $S_{3}$, yet the cycle only has length 2 . This happens because we select disjoint cycles from the group generators instead of the group elements themselves to avoid listing all permutations of a group. At the moment we let GAP select the generators automatically, but an improved implementation should take care of selecting generators having long cycles.

\section{Conclusion and future work}

We proposed an automatic way to compute permutations of the symmetry group of a MILP in general form (1) and derived two types of global symmetry breaking constraints designed to reduce the number of symmetric solutions. We exhibited a few preliminary experimental results indicating a positive trend. Future work will concentrate on: (i) reducing the computational effort taken to find permutations by means of exploitation of the block structure of the MILP constraint matrix; (ii) finding a method to reduce the influence of the arbitrary choice of orbit representative in (6), (7) not based on adding variables to the problem; (iii) extend the computational results to a more significant instance test set.

\section{References}

1. D. Bell. Constructive group relaxations for integer programs. SIAM Journal on Applied Mathematics, 30(4):708-719, 1976.

2. M. Boulle. Compact mathematical formulation for graph partitioning. Optimization and Engineering, 5:315-333, 2004.

3. D. Cohen, P. Jeavons, C. Jefferson, K. Petrie, and B. Smith. Symmetry definitions for constraint satisfaction problems. In P. van Beek, editor, $C P$, volume 3709 of LNCS. Springer, Berlin, 2005.

4. R. Fourer and D. Gay. The AMPL Book. Duxbury Press, Pacific Grove, 2002. 
5. E.J. Friedman. Fundamental domains for integer programs with symmetries. In A. Dress, Y. Xu, and B. Zhu, editors, COCOA, volume 4616 of LNCS, pages 146153, Berlin, 2007. Springer.

6. The GAP Group. GAP - Groups, Algorithms, and Programming, Version 4.4.10, 2007.

7. R. Gomory. Some polyhedra related to combinatorial problems. Linear Algebra and Its Applications, 2(4):451-558, 1969.

8. ILOG. ILOG CPLEX 10.1 User's Manual. ILOG S.A., Gentilly, France, 2006.

9. V. Kaibel and M. Pfetsch. Packing and partitioning orbitopes. Mathematical Programming, To appear.

10. J. Lee. All-different polytopes. Journal of Combinatorial Optimization, 6:335-352, 2002.

11. J. Lee and F. Margot. On a binary-encoded ILP coloring formulation. INFORMS Journal on Computing, 19(3):406-415, 2007.

12. L. Liberti. Reformulation techniques in mathematical programming, November 2007. Thèse d'Habilitation à Diriger des Recherches.

13. L. Liberti. Reformulations in mathematical programming: Definitions. In R. Aringhieri, R. Cordone, and G. Righini, editors, Proceedings of the 7th CologneTwente Workshop on Graphs and Combinatorial Optimization, Crema, 2008. Università Statale di Milano.

14. F. Margot. Pruning by isomorphism in branch-and-cut. Mathematical Programming, 94:71-90, 2002.

15. F. Margot. Exploiting orbits in symmetric ILP. Mathematical Programming B, 98:3-21, 2003.

16. F. Margot. Small covering designs by branch-and-cut. Mathematical Programming B, 94:207-220, 2003.

17. F. Margot. Symmetric ILP: coloring and small integers. Discrete Optimization, 4:40-62, 2007.

18. A. Martin, T. Achterberg, and T. Koch. MIPLIB 2003. A library of pure and mixed-Integer programs.

19. G.L. Nemhauser and L.A. Wolsey. Integer and Combinatorial Optimization. Wiley, New York, 1988.

20. J. Ostrowski, J. Linderoth, F. Rossi, and S. Smriglio. Orbital branching. In M. Fischetti and D.P. Williamson, editors, IPCO, volume 4513 of LNCS, pages 104-118, Berlin, 2007. Springer.

21. H. Sherali and C. Smith. Improving discrete model representations via symmetry considerations. Management Science, 47(10):1396-1407, 2001.

22. L. Wolsey. Group representation theory in integer programming. Technical Report Op. Res. Center 41, MIT, 1969.

\section{Appendix}

- Proof of Prop. 2.4. Let $\pi \in G_{P}$ and suppose $\exists x^{*} \in \mathcal{G}(P)\left(x^{*} \pi \notin G^{*}\right)$. Then either $x^{*} \pi$ is feasible in $P$ but not optimal, or it is infeasible. In the former case, $c^{\top}\left(x^{*} \pi\right)=\sum_{j \leq n} c_{j} x_{\pi_{(j)}^{*}}^{*}=\sum_{\pi^{-1}(j) \leq n} c_{\pi^{-1}(j)} x_{j}^{*}=\left(\pi^{-1} c^{\top}\right) x^{*}$, so necessarily $c \pi^{-1} \neq c$, which contradicts $\pi \in G_{P}$. In the latter case, suppose $\sigma A x^{*} \pi \not \leq \sigma b$ for all $\sigma \in S_{m}$, then a computation on indices (similar to the one above) implies $\forall \sigma \in S_{m}\left(\sigma A \pi x^{*} \not \subset \sigma b\right)$, which also contradicts $\pi \in G_{P}$ 
(the other infeasibility cases $x^{*} \notin\left[x^{L}, x^{U}\right]$ or $x_{j}^{*}$ is fractional for some $j \in Z$ are similar).

- Proof of Prop. 2.5. The map $\varphi(x[\sigma])=\bar{\chi}\left(x[\sigma]-x^{L}[\sigma]\right)$ is injective on $X^{\prime}=\left[x_{\sigma_{1}}^{L}, x_{\sigma_{1}}^{U}\right]^{k}$ by definition, as it makes $x[\sigma]-x^{L}[\sigma]$ the base- $\chi$ arithmetic representation for $\varphi(x[\sigma])$. For all $Y \in \mathcal{E}(\langle\sigma\rangle, \bar{X})$ we have $Y \subseteq X^{\prime}$, so the injectivity of $\varphi$ implies that there is a unique element $x^{\prime}$ of $Y$ such that $\varphi\left(x^{\prime}\right)$ is minimum. Therefore the constraints

$$
\forall\left(y \neq x^{\prime}\right) \in Y \quad(\varphi(x) \leq \varphi(y))
$$

are only satisfied by $x=x^{\prime}$. Furthermore, since $Y=x\langle\sigma\rangle$ for any $x \in Y$, the quantification of (22) can be written as $\forall 1 \leq h \leq k-1$, with $y$ in (22) replaced by $x \sigma^{h}$. The result follows.

- Proof of Prop. 2.6. Let $y^{\prime} \in Y=x\langle\sigma\rangle \in \mathcal{E}(\langle\sigma\rangle, \bar{X}), h \in\{1, \ldots, k\}$ such that $y_{\sigma^{k-h}(1)}^{\prime}=\min \operatorname{ran}\left(y^{\prime}\right)$, and $y=y^{\prime} \sigma^{h}$. Then obviously $y$ satisfies $(7)$. By definition of $y$ and its range, for all $h \in\{2, \ldots, \ell\}$ there is $j(h) \leq k$ such that $y_{\sigma_{k-j(h)}}>y_{\sigma_{1}}$, therefore $y \sigma^{j(h)}$ does not satisfy $(7)$. Notice that since $\ell \leq k$, the map $j$ can be chosen so that it is injective, $y \sigma^{j(h)}$ are all distinct for $2 \leq h \leq \ell$; hence, there are at least $\ell-1$ distinct elements of $Y$ not satisfying $(7)$.

- Proof of Prop. 3.1. Notice that $\Gamma(P)$ is never empty, because it always contains $\left(I_{m}, I_{n}\right)$. Since for any $(\sigma, \pi) \in \hat{G}_{P}$ such that $\pi \neq e,(\vartheta(\sigma), \vartheta(\pi))$ is feasible in $(17), \mathcal{F}\left(\operatorname{FPP}\left(P, j^{\prime}\right)\right)=\Gamma(P) \backslash\left\{\left(I_{m}, I_{n}\right)\right\}$, which implies the result. 